\title{
Antioxidative activities of alpha-mangostin in high-fat/high-glucose diet and streptozotocin-induced insulin-resistant rodents
}

\author{
Gilbert Lazarus, Sean Alexander, Gabrielle Ophelia Kusuma, Kevin Wijaya, Vivian Soetikno* \\ Department of Pharmacology and Therapeutics, Faculty of Medicine, Universitas Indonesia, Jakarta 10430, Indonesia.
}

\section{ARTICLE INFO \\ Received on: $11 / 05 / 2020$ \\ Accepted on: 17/07/2020 \\ Available online: 05/11/2020}

\section{Key words:}

Stress, oxidative, antioxidants, alpha-mangostin, insulin sensitivity.

\begin{abstract}
Insulin resistance (IR), of which pathomechanism is mainly mediated by oxidative stress, leads to impaired insulin secretion and multisystem damages. Recent evidence suggests that alpha-mangostin ( $\alpha$-MG) is a potential candidate to ameliorate oxidative stress; hence, this study aims to evaluate the antioxidative properties of $\alpha$-MG in IR-induced rats. A total of 36 male Wistar rats were divided into six groups, i.e., control, control $+\alpha-M G(200 \mathrm{mg} / \mathrm{kg}), \mathrm{IR}, \mathrm{IR}$ + metformin, and IR + various doses of $\alpha-M G(100$ and $200 \mathrm{mg} / \mathrm{kg}$ ) which were administered via oral gavage for 8 weeks. IR was induced by administering high-fat/high-glucose diet for 11 weeks followed by a single streptozotocin injection (35 mg/kg, i.p.). Malondialdehyde (MDA), superoxide dismutase (SOD), and glutathione (GSH) activities were assayed to assess the oxidative stress between groups. Welch's analysis of variance and Kruskal-Wallis test were used to compare the data. This study demonstrated that $\alpha-M G$ remarkably decreased MDA and increased SOD as well as GSH activities in the heart, liver, and kidney tissues of IR model. Furthermore, $\alpha$-MG also upregulated SOD activity in heart tissues in a dose-dependent manner. Besides its antioxidative effects, the administration of $\alpha$-MG at $200 \mathrm{mg} / \mathrm{kg}$ was also associated with the upregulation of skeletal glucose transporter type 4 expression $(2.50 \pm 0.43 \mathrm{vs}$. control, $3.65 \pm 0.36 \mathrm{ng} / \mathrm{ml} ; p<0.05)$. These findings suggest that $\alpha-\mathrm{MG}$ yielded the potent antioxidative properties against IR rats.
\end{abstract}

\section{INTRODUCTION}

Type 2 diabetes mellitus as caused by insulin resistance (IR) is the ninth leading cause of death, afflicting approximately one-tenth of adult population worldwide. The high mortality rate of this disease may be explained by the amplified risk of multisystemic complications, including cardiovascular disease, stroke, nephropathy, retinopathy, and neuropathy (Zheng et al., 2018). The pathomechanism of this disease involves impairment in insulin secretion and/or action as characterized by decreased glucose transporter type 4 (GLUT4) expression, both of which are mediated by oxidative stress (Asmat et al., 2016). Oxidative damages are evident in IR, illustrated by a decreased antioxidant protection including superoxide dismutase

\section{"Corresponding Author}

Vivian Soetikno, Department of Pharmacology and Therapeutics, Faculty of Medicine, Universitas Indonesia, Jakarta 10430, Indonesia.

E-mail: vivian.soetikno@ui.ac.id
(SOD) and glutathione (GSH) (Asmat et al., 2016), as well as increased malondialdehyde (MDA) levels (Tiwari et al., 2013). Oxidative stress in IR is mainly associated with cellular injury and endothelial dysfunction, which play major roles in the development of cardiovascular diseases through DNA, lipid, and protein damages (Asmat et al., 2016).

The treatment of IR involves blood glucose control through pharmacotherapies as well as lifestyle and diet changes (Ladeiras-Lopes et al., 2015). However, several concerns regarding the use of pharmacotherapies, particularly allopathic drugs, have been reported as they may induce severe adverse effects leading to treatment discontinuation (Karimi et al., 2015). Hence, the recent development of IR treatments has favored herbal medicines, which may yield similar efficacy with potentially fewer side effects (WHO, 1980). Alpha-mangostin ( $\alpha-M G)$, a major mangostin-purified xanthone, is known for its antioxidative properties by scavenging free radicals, inhibiting apolipoprotein B-100 oxidation, and ultimately preventing cellular deaths $(\mathrm{Xu}$ et al., 2017). Furthermore, research works have also shown that $\alpha$-MG yields potent anti-inflammatory, anticancer, antimicrobial, 
and antiplasmodial activities (Asasutjarit et al., 2019; Ibrahim et al., 2016; Larson et al., 2010). Although recent evidence suggests that $\alpha-M G$ yielded potent antioxidative potentials, little is known regarding its effects on the oxidative markers of various organs and skeletal GLUT4 expressions in IR. Hence, this study aims to evaluate the effects of $\alpha-\mathrm{MG}$ in oxidative stress and skeletal GLUT4 expressions in IR model rats.

\section{MATERIALS AND METHODS}

\section{Animals}

A total of 36 male Wistar rats with age ranging from 10 to 12 months (150-250 g) were bred and handled at the Institute of Health Research and Development, Faculty of Medicine, Universitas Indonesia, Jakarta. This study's protocol was approved by the Institute of Animal Studies Ethics Committee (Guo et al., 2018). Animals were housed in standard condition (12 hours light/ dark cycle; $23^{\circ} \mathrm{C} \pm 2{ }^{\circ} \mathrm{C}$ ) and were provided with ad libitum access to food and water.

\section{Study protocol}

The details on experiment procedures have been previously discussed elsewhere (Soetikno et al., 2020). In brief, the animals were randomly allocated to the following six groups of six rats each: (1) normal group, serving as control (C); (2) healthy rats supplemented by $\alpha-\mathrm{MG} 200 \mathrm{mg} / \mathrm{kg}(\mathrm{C}+\alpha-\mathrm{MG} 200)$ (Aktin Chemicals Inc., Chengdu, China); (3) untreated IR-induced rats; (4) IR-induced rats treated with metformin $200 \mathrm{mg} / \mathrm{kg}$ (IR + metformin); (5) IR-induced rats treated with $\alpha$-MG $100 \mathrm{mg} / \mathrm{kg}$ (IR $+\alpha$-MG 100); and (6) IR-induced rats treated with $\alpha-M G 200 \mathrm{mg} /$ $\mathrm{kg}$ (IR $+\alpha$-MG 200). Experimental rats were induced with IR by ad libitum high-fat/high-glucose [high-fat/high-glucose (HF/HG), high-fat diet (HFD), $46.1 \%$ energy as fat, $18.1 \%$ protein, and $35.8 \%$ carbohydrate, 58V8, TestDiet, St. Louis, MO] diet for 11 weeks as well as single intraperitoneal streptozotocin [streptozotocin (STZ), 35 mg/kg, Santa Cruz, Dallas, TX; catalog number sc-200719A] injection at week 3 . On sacrifice, tissue samples were excised and stored at $-80^{\circ} \mathrm{C}$ until further measurements.

\section{Homeostasis model assessment-IR (HOMA-IR) index measurement}

The HOMA index was measured using the steadystate blood glucose level along with insulin concentrations and was calculated using the following formula: HOMA-IR = blood glucose concentration $(\mathrm{mM}) \times$ insulin $(\mu \mathrm{U} / 1) / 22.5$.

\section{Oxidative stress marker assays}

The following oxidative stress markers were assessed in this study: MDA, SOD, and GSH. MDA level was measured using lipid peroxidation assay kit (Sigma-Aldrich, Singapore; catalog number MAK085), SOD activity using colorimetric activity kit (Invitrogen, Carlsbad, CA; catalog number EIASODC), and GSH level with colorimetric detection kit (Invitrogen, Carlsbad, CA; catalog number EIAGSHC), all of which were performed to the manufacturers' instructions. The obtained concentration for each oxidative stress marker was normalized for tissue protein content, and MDA level and GSH and SOD concentrations were expressed as $\mathrm{nmol} / \mathrm{mg}$ protein, $\mu \mathrm{mol} / \mathrm{mg}$ protein, and $\mathrm{U} / \mathrm{mg}$ protein, respectively.

\section{Estimation of GLUT4 expression in homogenized skeletal muscle}

The effects of $\alpha-M G$ on GLUT4 expression in skeletal muscle tissue were analyzed by using the sandwich-ELISA kit for GLUT4 (Cusabio, Houston, TX; catalog number CSB-E13908r), carried out per manufacturer's instructions.

\section{Statistical analysis}

The findings are presented as mean \pm standard deviation (SD) or as median (interquartile range (IQR)), whichever appropriate. The distribution and homogeneity of the data were analyzed with the Shapiro-Wilk test and Levene's test, respectively. Normally distributed and homogenous data were analyzed using the one-way analysis of variance (ANOVA) followed by Tukey's HSD test which was used to compare significance among groups, whereas the analysis of normal heteroscedastic data was performed with Welch's ANOVA followed by Games-Howell post hoc test. Otherwise, Kruskal-Wallis H-test followed by Mann-Whitney U post hoc test was utilized. All statistical analyses were performed using the Statistical Package for the Social Sciences 21.0 (IBM, Armonk, NY) and GraphPad Prism (version 8.4.2), and a $p$-value of $<0.05$ was considered to be statistical significance.

\section{RESULTS}

\section{Effects of $\alpha$-MG on body weight and HOMA-IR}

Following $\mathrm{HF} / \mathrm{HG} / \mathrm{STZ}$ induction, the mean body weight of the IR group was lower than of other groups. Although $\alpha-\mathrm{MG}$ administration did not show changes in the body weight of control group, its administration was able to prevent weight loss within the IR groups, and this effect was not significantly different among groups (Fig. 1A). The measurement of HOMA-IR revealed

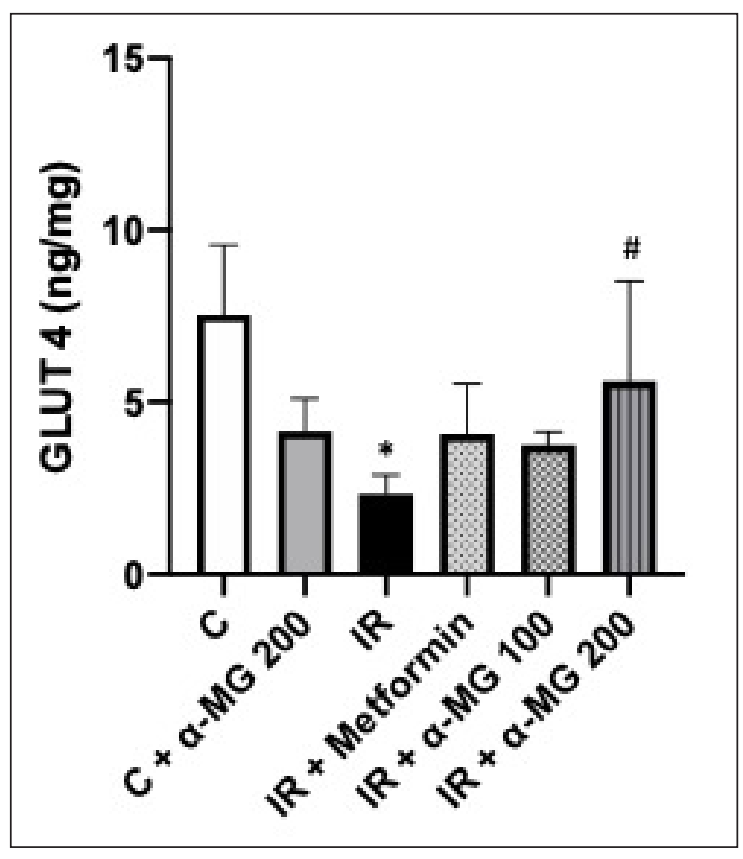

Figure 2. Effect of alpha-mangostin in both doses (100 and $200 \mathrm{mg} / \mathrm{kg})$ and metformin on GLUT4 expression in the skeletal muscle of HF/HG/STZ-induced IR rats. Data were analyzed with one-way ANOVA using GraphPad Prism software and presented in mean $\pm \mathrm{SD}(n=6) .{ }^{*} p<0.05$ versus $\mathrm{C}$ and $\mathrm{C}+\alpha$-MG 200. ${ }^{*} p<0.05$ versus IR. 
that the administration of $\alpha-\mathrm{MG}$ in both doses (i.e., 100 and 200 $\mathrm{mg} / \mathrm{kg}$ ) significantly increased insulin sensitivity, evident in the decreased HOMA-IR index when compared to untreated IR group (Fig. 1B).

\section{Effects of $\alpha$-MG on MDA levels}

In this study, we demonstrated that oxidative stress ensues after HF/HG/STZ-induced IR, as shown in Table 1, where MDA concentration was higher in IR-induced diabetic rats than in control. The administration of $\alpha-\mathrm{MG}$ in both doses on the heart and kidney tissues resulted in decreased MDA levels when compared to IR groups. Although the administration of $\alpha-\mathrm{MG}$ at both 100 and $200 \mathrm{mg} / \mathrm{kg}$ resulted in significantly lower MDA concentrations, the desired effect was not observed in a dose-dependent manner. Nonetheless, the administration of $\alpha-\mathrm{MG}$ at $200 \mathrm{mg} / \mathrm{kg}$ in heart tissues substantially ameliorated MDA concentrations better than metformin; however, this was not the case for kidney and liver tissues.

\section{Effects of $\alpha$-MG on antioxidants activity}

The upregulation of the activities of antioxidant enzymes (i.e., SOD and GSH) was also observed with the administration of $\alpha$-MG. Alpha-mangostin $200 \mathrm{mg} / \mathrm{kg}$ successfully ameliorated SOD activities in heart and kidney tissues $(p<0.001)$, whereas its improvement following $\alpha-\mathrm{MG}$ administration at $100 \mathrm{mg} /$ $\mathrm{kg}$ was only significant for heart tissues $(p<0.001)$. Similarly, the administration of $\alpha-\mathrm{MG}$ at both 100 and $200 \mathrm{mg} / \mathrm{kg}$ also upregulated GSH activities (both at $p=0.029$ ) (Table 1).

\section{Effects of $\alpha$-MG on GLUT4 expression}

In terms of GLUT4 expression, the downregulation of GLUT4 expression in the skeletal muscle tissues following IR induction was also observed $(2.50 \pm 0.43 \mathrm{ng} / \mathrm{ml})$, as compared to control $(9.24 \pm 2.24 \mathrm{ng} / \mathrm{ml}, p=0.039)$. Subsequently, the administration of $\alpha-\mathrm{MG}$ at $200 \mathrm{mg} / \mathrm{kg}$ significantly improved GLUT4 expression ( $p=0.045$ ) (Fig. 2).

\section{DISCUSSION}

The correlation between oxidative stress and IR has been well established. The reciprocal amplification between these two components may lead to deteriorations in body function and quality of life. While it may be true that oxidative stress is the root cause of IR, it is also the leading cause of pathophysiological changes in IR, including reduced nitric oxide as well as increased inflammatory cytokines and acute-phase reactants, which may lead to massive glycation and oxidation resulting in multiorgan failure, including atherosclerosis and major adverse cardiac events, kidney failure, and severe liver disorders (Wright et al., 2006), therefore, intensifying the need to develop alternative modalities to ameliorate oxidative stress and reduce its disease burden.

This study demonstrated that $\alpha-\mathrm{MG}$ yielded protective effects against oxidative stress and GLUT4 expression in IRinduced rats. Although the antioxidative properties of $\alpha-M G$ are well established, to the best of authors' knowledge, its effect on skeletal GLUT4 expression as well as its potential antioxidative activities against IR, particularly on heart and liver tissues, has yet to be investigated. Overall, $\alpha-\mathrm{MG}$ improved oxidative stress in diabetic organs, evident in the increase of SOD and GSH activities as well as decrease in MDA level. Its antioxidative effect on heart tissues was better augmented following the administration of $\alpha-\mathrm{MG}$ $200 \mathrm{mg} / \mathrm{kg}$. However, this was not the case for kidney and liver tissues, where the administration of $\alpha-\mathrm{MG} 200 \mathrm{mg} / \mathrm{kg}$ resulted in lesser improvements of oxidative stress when compared to those of $100 \mathrm{mg} / \mathrm{kg}$ although insignificant, which may be explained by the different responses of various organs to oxidative stress and antioxidative mechanism (Liu et al., 2000).

The results corroborated findings of the previous studies, where $\alpha-\mathrm{MG}$ exhibited cardioprotective effects on isoproterenolinduced myocardial infarction (Sampath et al., 2007), renoprotective effects on cisplatin-induced nephrotoxicity (PérezRojas et al., 2009), and hepatoprotective effects on acute liver failure (Fu et al., 2018). The antioxidative properties of $\alpha$-MG are mediated by its phenol-rich content, which may act as a free radical

Table 1. Study outcomes.

\begin{tabular}{|c|c|c|c|c|c|c|}
\hline Variable & $\begin{array}{c}\text { C } \\
(n=6)\end{array}$ & 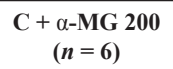 & $\begin{array}{c}\text { IR } \\
(n=6)\end{array}$ & $\begin{array}{c}\text { IR + metformin } \\
(n=6)\end{array}$ & $\begin{array}{c}\text { IR }+\alpha-M G 100 \\
(n=6)\end{array}$ & $\begin{array}{c}\text { IR }+\alpha-\text { MG } 200 \\
(n=6)\end{array}$ \\
\hline \multicolumn{7}{|l|}{ MDA (nmol/mg) } \\
\hline Heart & $2.29 \pm 0.78$ & $2.44 \pm 0.50$ & $6.46 \pm 1.03 *$ & $2.44 \pm 0.36^{* *}$ & $2.05 \pm 0.23^{* *}$ & $\pm 0.15^{* *}$ \\
\hline Liver & $0.24 \pm 0.00$ & $0.02 \pm 0.01$ & $0.078 \pm 0.05^{*}$ & $0.05 \pm 0.02$ & $0.04 \pm 0.01$ & \pm 0.01 \\
\hline Kidney & $0.11 \pm 0.52$ & $0.09 \pm 0.03$ & $0.92 \pm 0.33^{*}$ & $0.05 \pm 0.02 * *$ & $0.15 \pm 0.08^{* *}$ & $0.26 \pm 0.10^{* *}$ \\
\hline \multicolumn{7}{|l|}{$\mathrm{SOD}(\mathrm{U} / \mathrm{ml})$} \\
\hline Heart & $1.06 \pm 0.15$ & $1.39 \pm 0.36$ & $0.14 \pm 0.08 *$ & $1.61 \pm 0.35^{* *}$ & $2.01 \pm 0.17^{* *}$ & $\pm 0.29 * *$ \\
\hline Kidney & $0.61 \pm 0.27$ & $0.26 \pm 0.18$ & $0.21 \pm 0.07 *$ & $0.29 \pm 0.12$ & $0.45 \pm 0.18$ & $0.54 \pm 0.16^{* *}$ \\
\hline \multicolumn{7}{|l|}{$\mathrm{GSH}(\mu \mathrm{M})$} \\
\hline Liver & $0.36 \pm 0.06$ & $0.35 \pm 0.23$ & $0.26 \pm 0.04 *$ & $0.30 \pm 0.01 * *$ & $0.36 \pm 0.11^{* *}$ & $0.29 \pm 0.04 * *$ \\
\hline Skeletal GLUT4 (ng/ml) & $9.24 \pm 2.24$ & $4.40 \pm 0.10$ & $2.50 \pm 0.43^{*}$ & $3.63 \pm 0.29 * *$ & $6.11 \pm 2.22$ & $3.65 \pm 0.36^{* *}$ \\
\hline
\end{tabular}

Results are presented as mean $\pm \mathrm{SD}$ or median (IQR), whichever appropriate. Values are considered statistically significant at $p<0.05 . \mathrm{AM}=$ alpha-mangostin; $\mathrm{HF} / \mathrm{HG} / \mathrm{STZ}$ $=$ high-fat/high-glucose diet + streptozotocin injection i.p.; MDA = malondialdehyde; $\mathrm{SOD}=$ superoxide dismutase; GSH = glutathione; GLUT4 = glucose transporter type 4 . $* p<0.05$ versus control. $* * p<0.05$ versus IR. 


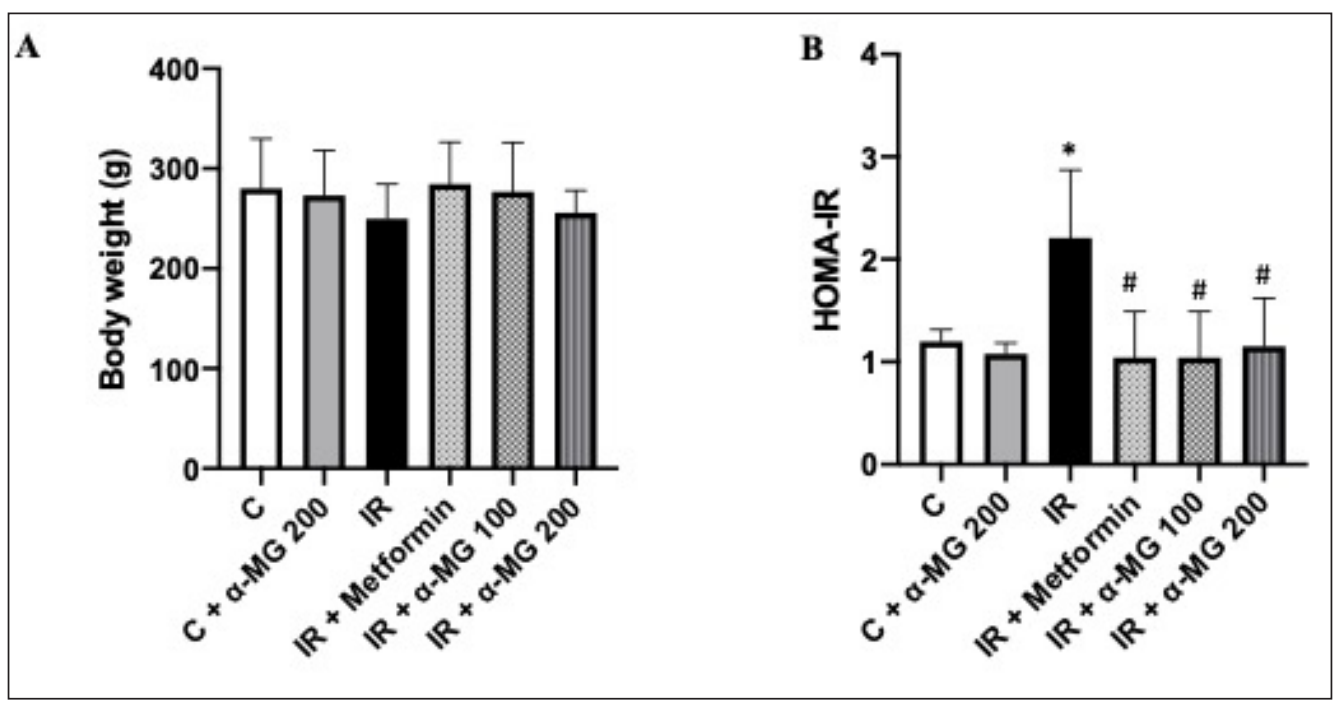

Figure 1. Effect of alpha-mangostin in both doses $(100$ and $200 \mathrm{mg} / \mathrm{kg}$ ) and metformin on body weight (A) and HOMAIR (B) in HF/HG/STZ-induced IR rats. Data were analyzed with one-way ANOVA using GraphPad Prism software and presented in mean $\pm \mathrm{SD}(n=6) .{ }^{*} p<0.05$ versus $\mathrm{C}$ and $\mathrm{C}+\alpha-\mathrm{MG} 200 .{ }^{*} p<0.05$ versus IR.

scavenger as well as a modulator for several antioxidant enzymes (Ibrahim et al., 2016). Furthermore, $\alpha$-MG may also downregulate peroxisome proliferator-activated receptor- $\gamma$ expression, thereby inhibiting adipo/lipogenesis on 3T3-L1 cells and subsequently reducing lipid peroxidation (Taher et al., 2015).

The supplementation of $\alpha$-MG on healthy rats did not yield positive results. On the contrary, it even reduced SOD and GSH activities on renal and liver tissues, respectively. This may be explained by the potentials of -MG to act as a prooxidant in oxidative balance state. Kosem et al. (2013) stated that the overdose of $\alpha-\mathrm{MG}$ may induce severe hepatotoxicity and renotoxicity. Furthermore, the administration of high-dose $\alpha-\mathrm{MG}$ may also cause hypoactivity, lethargy, decreased appetite, and piloerection, which were observed in this study.

In addition to the improvements in oxidative stress, $\alpha-M G$ was also associated with increased skeletal GLUT4 expression, suggesting that $\alpha-\mathrm{MG}$ may also yield antidiabetic effects. This is, especially, true as skeletal GLUT4 expression was severely diminished in T2DM patients, thus worsening IR and the disease progression (Shan et al., 2011). The antidiabetic property of $\alpha-\mathrm{MG}$ observed in this study may be related to its ability to stimulate insulin secretion by activating the insulin receptor and to inhibit the phosphorylation of insulin receptor substrate-1 (Lee et al., 2018). This study added that, besides increased GLUT4 expression in adipocyte (Taher et al., 2015), liver (Kim et al., 2017), and cardiac tissues (Ratwita et al., 2017), $\alpha-M G$ was also able to upregulate GLUT4 expression in skeletal muscles.

In this study, we used experimental animals that were administered with high-fat diet for 11 weeks and a single lowdose STZ injection at week 3 to induce IR. The method that we used is able to cause IR in all experimental animals, and this is in accordance with research conducted by Al-Trad et al. (2019) who analyzed the effects of eugenol on oxidative stress and inflammatory processes in rats with T2DM. IR which is commonly followed by obesity can be induced in experimental animals by providing a high-fat diet for several weeks to months. However, there are several studies that prove that IR can be detected in rodents only after 1-3 weeks of high-fat diet before an increase in body mass (Castorena et al., 2014; Hancock et al., 2008; Turner et al., 2013).

\section{CONCLUSION}

To summarize, this study confirmed the antioxidative properties of $\alpha-\mathrm{MG}$ on heart, liver, and kidney tissues, evident in the decreased MDA levels as well as increased SOD and GSH activities. Furthermore, $\alpha-\mathrm{MG}$ was also capable of upregulating skeletal GLUT4 expression, yielding potent antidiabetic effects in addition to its antioxidative activities. Future studies investigating the molecular mechanism of $\alpha-\mathrm{MG}$ are required to better elucidate our findings.

\section{ACKNOWLEDGMENTS}

The authors would like to express their gratitude to all biotechnicians of the Clinical Pharmacology Laboratory and Integrated Laboratory, Universitas Indonesia, for their tremendous help and support in the conduction of this study. The authors also acknowledge the Directorate Research and Community Services of Universitas Indonesia for the provision of financial supports.

\section{CONFLICT OF INTEREST}

Authors declared that they do not have any conflicts of interest.

\section{FUNDING}

None.

\section{REFERENCES}

Al-Trad B, Alkhateeb H, Alsmadi W, Al-Zoubi M. Eugenol ameliorates insulin resistance, oxidative stress and inflammation in high fat-diet/streptozotocin-induced diabetic rat. Life Sci, 2019; 216:183-8;

Asasutjarit R, Meesomboon T, Adulheem P, Kittiwisut S, Sookdee P, Samosornsuk W, Fuongfuchat A. Physicochemical properties 
of alpha-mangostin loaded nanomeulsions prepared by ultrasonication technique. Heliyon, 2019; 5(9):e02465.

Asmat U, Abad K, Ismail K. Diabetes mellitus and oxidative stress-a concise review. Saudi Pharm J, 2016; 24(5):547-53.

Castorena CM, Arias EB, Sharma N, Cartee GD. Postexercise improvement in insulin-stimulated glucose uptake occurs concomitant with greater AS160 phosphorylation in muscle from normal and insulin-resistant rats. Diabetes, 2014; 63(7):2297-308;

Fu T, Li H, Zhao Y, Cai E, Zhu H, Li P, Liu J. Hepatoprotective effect of alpha-mangostin against lipopolysaccharide/d-galactosamine-induced acute liver failure in mice. Biomed Pharmacother, 2018; 106:896-901.

Guo X-X, Wang Y, Wang K, Ji BP, Zhou F. Stability of a type 2 diabetes rat model induced by high-fat diet feeding with low-dose streptozotocin injection. J Zhejiang Univ Sci B, 2018; 19(7):559-69.

Hancock CR, Han DH, Chen M, Terada S, Yasuda T, Wright DC, Holloszy JO. High-fat diets cause insulin resistance despite an increase in muscle mitochondria. Proc Natl Acad Sci U S A, 2008; 105(22):7815-20;

Ibrahim MY, Hashim NM, Mariod AA, Mohan S, Abdulla MA, Abdelwahab SI, Arbabe IA. $\alpha$-mangostin from Garcinia mangostana Linn: an updated review of its pharmacological properties. Arab J Chem, 2016; 9(3): 317-29

Karimi A, Majlesi M, Rafieian-Kopaei M. Herbal versus synthetic drugs; beliefs and facts. J Nephropharmacol, 2015; 4(1):27-30.

Kim HM, Kim YM, Huh JH, Lee ES, Kwon MH, Lee BR, Ko $\mathrm{H}-\mathrm{J}$, Chung CH. $\alpha$-mangostin ameliorates hepatic steatosis and insulin resistance by inhibition C-C chemokine receptor 2. PLoS One, 2017; 12(6): 0179204.

Kosem N, Ichikawa $\mathrm{K}$, Utsumi $\mathrm{H}$, Moongkarndi $\mathrm{P}$. In vivo toxicity and antitumor activity of mangosteen extract. J Nat Med, 2013; 67(2):255-63.

Ladeiras-Lopes R, Fontes-Carvalho R, Bettencourt N, Sampaio F, Gama V, Leite-Moreira A. Novel therapeutic targets of metformin: metabolic syndrome and cardiovascular disease. Expert Opin Ther Targets, 2015; 19(7):869-77.

Larson RT, Lorch JM, Pridgeon JW, Becnel JJ, Clark GG, Lan Q. The biological activity of alpha-mangostin, a larvicidal botanic mosquito sterol carrier protein-2 inhibitor. J Med Entomol, 2010; 47(2):249-57.

Lee D, Kim Y-M, Jung K, Chin Y-W, Kang K. Alpha-mangostin improves insulin secretion and protects INS-1 cells from streptozotocininduced damage. Int J Mol Sci, 2018; 19(5):1484.

Liu J, Yeo HC, Overvik-Douki E, Hagen T, Doniger SJ, Chyu DW, Brooks GA, Ames BN. Chronically and acutely exercised rats: biomarkers of oxidative stress and endogenous antioxidants. J Appl Physiol, (1985). 2000; 89(1): 21-8.

Pérez-Rojas JM, Cruz C, García-López P, Sánchez-González DJ, Martínez-Martínez CM, Ceballos G, Espinosa M, Meléndez-Zajgla J, Pedraza-Chaverri J. Renoprotection by alpha-mangostin is related to the attenuation in renal oxidative/nitrosative stress induced by cisplatin nephrotoxicity. Free Radic Res, 2009; 43(11):1122-32.

Ratwita SE, Sukandar EY, Adnyana IK, Kurniati NF. Alpha mangostin and xanthone from mangosteen (Garcinia mangostana 1.) role on glucose tolerance and glucose transporter-4 in diabetes mellitus. Int J Pharmacogn Phytochem Res, 2017; 9(9):1206-12.

Sampath PD, Vijayaraghavan K. Cardioprotective effect of $\alpha$-mangostin, a xanthone derivative from mangosteen on tissue defense system against isoproterenol-induced myocardial infarction in rats. $\mathrm{J}$ Biochem Mol Toxicol, 2007; 21(6):336-9.

Shan W-F, Chen B-Q, Zhu S-J, Jiang L, Zhou Y-F. Effects of GLUT4 expression on insulin resistance in patients with advanced liver cirrhosis. J Zhejiang Univ Sci B, 2011; 12(8):677-82.

Soetikno V, Andriyani A, Andini P, Charlie F, Lazarus G, Louisa M, Arozal W. Alpha mangostin improves cardiac hypertrophy and fibrosis and associated biochemical parameters in high-fat/high-glucose diet and low dose streptozotocin injection-induced type 2 diabetic rats. J Exp Pharmacol, 2020; 12:27-38.

Taher M, Mohamed Amiroudine MZA, Tengku Zakaria TMFS, Susanti D, Ichwan SJA, Kaderi MA, Ahmed QU, Zakaria ZA. $\alpha$-mangostin improves glucose uptake and inhibits adipocytes differentiation in 3T3-L1 cells via PPAR $\gamma$, GLUT4, and leptin expressions. Evid Based Complement Alternat Med, 2015; 2015:740238.

Tiwari BK, Pandey KB, Abidi AB, Rizvi SI. Markers of oxidative stress during diabetes mellitus. J Biomark, 2013; 2013:378790.

Turner N, Kowalski GM, Leslie SJ, Risis S, Yang C, Lee-Young RS, Babb JR, Meikle PJ, Lancaster GI, Henstridge DC, White PJ, Kraegen EW, Marette A, Cooney GJ, Febbraio MA, Bruce CR. Distinct patterns of tissue-specific lipid accumulation during the induction of insulin resistance in mice by high-fat feeding. Diabetologia, 2013; 56(7):1638-48;

WHO. Expert committee on diabetes mellitus: second report. World Health Organ Tech Rep Ser, 1980; 646:1-80.

Wright E. Jr, Scism-Bacon JL, Glass LC. Oxidative stress in type 2 diabetes: the role of fasting and postprandial glycaemia. Int J Clin Pract, 2006; 60(3):308-14.

$\mathrm{Xu}$ Y, Zhou H, Cai L. Alpha-mangostin attenuates oxidative stress and inflammation in adjuvant-induced arthritic rats. Trop J Pharm Res, 2017; 16:2611-6.

Zheng Y, Ley SH, Hu FB. Global aetiology and epidemiology of type 2 diabetes mellitus and its complications. Nat Rev Endocrinol, 2018; 14(2):88-98;

How to cite this article:

Lazarus G, Alexander S, Kusuma GO, Wijaya K, Soetikno V. Antioxidative activities of alpha-mangostin in high-fat/highglucose diet and streptozotocin-induced insulin-resistant rodents. J Appl Pharm Sci, 2020; 10(11):035-039. 